\title{
Immunogenicity to the bivalent HPV-16/18 vaccine among adolescent African students exposed to helminths and malaria
}

\author{
Miriam Nakalembe ${ }^{1}$, Cecily Banura ${ }^{2}$, Proscovia B Namujju ${ }^{3}$, Florence M Mirembe $^{1}$ \\ ${ }^{1}$ Department of Obstetrics and Gynaecology, Makerere University, Kampala, Uganda \\ ${ }^{2}$ Department of Child Health and Development Center, Makerere University, Kampala, Uganda \\ ${ }^{3}$ Department of Children's, Adolescent's and Adult's Health, National Institute for Health and Welfare, Oulu, Finland
}

\begin{abstract}
Introduction: Efficacious vaccines that prevent human papillomavirus (HPV) infection, the recognized cause of cervical cancer, are now available. However, in sub-Saharan Africa, immune-modulating infections such as helminths and malaria may affect immunogenicity to the HPV vaccine. This study aimed to evaluate the effect of helminth infections and exposure to malaria on the immune response to the bivalent HPV-16/18 vaccine.

Methodology:AS04-adjuvanted HPV-16/18 vaccinated students between 10 and 16 years of age from western Uganda, at 18 months-post vaccination were followed up for six months. After consent was obtained, demographic data, blood, and stool samples were collected. Multiplex HPV serology technology was used to determine HPV-16/18 antibody levels expressed as median fluorescent intensity (MFI). The malaria antibody immunoassay test was used to detect antibodies to malaria parasites. The Kato-Katz method was used to detect the presence of helminths. HPV-16/18 antibody levels among students exposed to malaria or helminths were compared with those who were not exposed using the Student's $t$-test.

Results: A total of 211 students participated in the study. There was no difference between MFI levels to HPV-16/18 antibodies at $18-$ and 24-month follow-ups among students who were positive and negative to malaria or helminth exposure. There was an increase in HPV-18 MFI antibody levels at month 24 among the students who were positive for malaria at enrolment $(\mathrm{p}=0.05)$.

Conclusions:Immune-modulating parasites (malaria/helminths) were not associated with reduced immune response to the bivalent HPV$16 / 18$ vaccine. The data may support the use of this vaccine in sub-Saharan Africa.
\end{abstract}

Key words: HPV vaccine; immunogenicity; helminths; malaria; Uganda.

J Infect Dev Ctries 2015; 9(2):197-205. doi:10.3855/jidc.5719

(Received 08 August 2014 - Accepted 19 November 2014)

Copyright $(2015$ Nakalembe et al. This is an open-access article distributed under the Creative Commons Attribution License, which permits unrestricted use, distribution, and reproduction in any medium, provided the original work is properly cited.

\section{Introduction}

Over $85 \%$ of the worldwide burden of cervical cancer occurs in the developing world, with Uganda bearing one of the highest incidence rates of the disease [1]. Human papillomavirus (HPV), the most common sexually transmitted infection, has been recognized as a cause of cervical cancer [2]. In Uganda, infection with high-risk HPV is common. Prevalence among both HIV-negative and -positive young women under 25 years of age ranges from $41.6 \%$ to $75.0 \%$ and $23.7 \%$ to $67.1 \%$, respectively, according to a recent review [3]. Therefore, prevention of HPV infection is a critical step in reducing the burden of cervical cancer.

The recently developed prophylactic vaccines against HPV infection are more than $90 \%$ effective in preventing infection and high-grade pre-cancer lesions associated with the HPV types included in the vaccine
[4]. However, in sub-Saharan Africa, prevalent immune-modulating infections such as malaria and helminths have been found to reduce the potency of some vaccines such as the bacillus Calmette-Guerin (BCG) and measles vaccine [5]. It has been suggested that helminth infection results in Th2-type cytokine secretion, high TGF- $\beta$ (transforming growth factor beta) levels, extremely low IFN- $\gamma$ secretion, and increased $\mathrm{CD}^{+} / \mathrm{CD} 25^{+} \mathrm{BT}$ regulatory cell population, altering the immunological balance [6]. The Th1 responses (required for protective immunity) are inhibited as a result of the immunological imbalance, consequently impairing responses to vaccines [6]. Similarly, repeated episodes of infections with malaria can also elicit immunological regulatory responses that may affect immune responses and effectiveness of vaccines [7]. During malaria infection, free heme that is released is toxic to the parasites. However, the 
parasites have developed a mechanism of detoxification by aggregating heme into hemozoin, an insoluble biomineral that protects the parasites from phagocytosis while maintaining other immunosuppressive activities.

Hence, parasites have the ability to downregulate the host immunity. The downregulation of the immune system may spill over, altering responses to vaccinations [5].

Therefore, through similar mechanisms of immune modulation, there is concern that the efficacy of the prophylactic HPV vaccines may be lower in tropical countries $[8,9]$. Hence, there is need to evaluate the effect of parasites such as malaria and helminths on the newly introduced HPV vaccines among the populations that are exposed to them [10].

Therefore, the objective of this study was to evaluate the level of immune response to the HPV$16 / 18$ vaccine among students who had evidence of exposure to malaria and helminth infections.

\section{Methodology}

Study site

The study was conducted in Ibanda, a district in western Uganda. Ibanda district covers an area of 967 square kilometers (373 sq mi). It consists of one county and seven sub-counties. It has an estimated population of 242,800 people. At the time of the study, Ibanda district had 180 primary schools with 23,695 students, of whom $6,770(28.5 \%)$ had been vaccinated against HPV-16/18 in April, May, and October 2010.

\section{Study design}

A prospective cohort study was conducted among 211 students who had been fully vaccinated (received three doses) with the AS04-adjuvanted HPV-16/18 vaccine beginning at 18 months (baseline results of antibodies published) [11] to 24 months postvaccination, between October 2011 and April 2012.

\section{Study population}

One administrative unit (sub-county) from Ibanda district was purposively selected. The selection was based on its population of at least 500 vaccinated students in primary level five, and its accessibility to the main road so that blood samples could easily be delivered daily to the laboratory (a distance of about $150 \mathrm{~km}$ ) for processing.

The selected sub-county had 29 schools, of which 15 (51\%) schools fulfilled the selection criteria. After permission from the district leadership to conduct the study was obtained, the head teachers of the selected schools were contacted by phone and briefly informed about the study and the letters they were about to receive from the community mobilizers. Community mobilizers, who were part of the study team, then visited the selected schools within the sub-county to deliver the letters that further explained the study, and requested permission from the head teachers to have their schools participate in the study. A convenient day for the study team to visit the school was agreed upon by both the school administration and the study team. The mobilizers used HPV vaccination registers from the district health office to verify HPV vaccination status. The head teachers were requested to invite parents/guardians of the students who had complete vaccination doses according to the register to come to the schools on the agreed-upon date. Trained research assistants and the principal investigator (PI) explained the study objectives and procedures to the students and their parents/guardians in separate sessions on the agreed-upon day of the school visits. After these sessions and responding to questions, the study staff obtained written informed consent from parents/guardians (guardians above 18 years of age). Finally, a written assent from the students who agreed to participate in the study and whose parents had consented was also obtained. All the students who were present on the day of the visit and had received three doses of the vaccine were eligible to participate. The students were consecutively recruited until the desired sample size was achieved.

\section{Study procedures}

At baseline (18 months post-vaccination), a standardized, interviewer-administered questionnaire bearing participant study numbers was used to obtain data on socio-demographic characteristics, vaccination status, and general medical and sexual history. Sexual activity was defined as having penetrative vaginal or anal sexual intercourse with the opposite sex. The students' names, serial numbers, study numbers, and school names were entered into a register for identification at their follow-up visits. In the meantime, through telephone contact, the head teachers of the participating schools were reminded to communicate to the students about the second visit and encouraged to be available on that date. At the 24month post-vaccination follow-up visit, the schools that had students who had initially participated in the study at baseline were revisited. The students were identified using an enrolment book that had the students' study numbers and names. Students who had changed to another participating school within the 
study period were identified by both their former school and name. However, students were considered lost to follow-up if they had changed to a nonparticipating school or were absent from school after three visits to the school.

Under aseptic conditions, blood samples were collected from each student at both 18 (baseline) and 24 months post-vaccination. A stool sample was collected once at baseline since all the girls were dewormed and a repeat de-worming was undertaken shortly before the 24-month follow-up visit.

\section{Blood sample collection}

Under aseptic procedure, $2-3 \mathrm{~mL}$ of blood was drawn from the cubital vein into a labelled serumseparating tube (SST)tube by the study staff. The vials of blood were sealed and put in transport boxes that had ice packs and transported to Mbarara University Epicenter laboratory within two to eight hours of collection. In the laboratory, the vials of blood were centrifuged at room temperature, after which $1 \mathrm{~mL}$ of serum was aliquoted and temporarily stored at $-80^{\circ} \mathrm{C}$ in Mbarara University Epicenter laboratory. Serum samples were then transported on dry ice to the molecular laboratory at the Makerere University College of Health Sciences and finally shipped on dry ice to Germany Cancer Research Center (DKFZ) in Heidelberg for HPV serological analysis.

\section{Serological evaluation of HPV antibodies}

Antibodies to the L1 proteins of HPV-16/18 were analyzed using a multiplex serology technology method. This method is based on a glutathione Stransferase capture immunosorbent assay combined with fluorescent-bead technology as described elsewhere [12-14].

\section{Procedure for estimating HPV antibodies}

Bead sets carrying different antigens were mixed and incubated with an equal volume of the serum dilutions $(2 \mu \mathrm{L})$ in a 96-well plate. Human antibodies bound to their respective antigens were stained with a secondary biotinylated goat anti-human immunoglobulin IgG (Dianova, Hamburg, Germany) and the fluorescent reporter conjugate streptavidin-Rphycoerythrin. A Luminex 100 Analyzer (Luminex, Austin, USA) determined the internal bead colour specific for the respective antigen and reporter fluorescence of the beads, reflecting the quantity of antibodies recognizing the respective antigens. For each bead set, the reporter fluorescence was expressed as median fluorescence intensity (MFI) of at least 100 beads per set per serum. Final antigen-specific MFI values were calculated by subtraction of glutathione Stransferase fusion proteins-tag (GST) and an individual bead background value. MFI levels of antibodies elicited by the HPV-16/18 vaccine were calculated as mean plus 3 standard deviations (SD) of sera from HPV-16/18 negative controls. The cut off level for positivity to HPV-16 was 373 while for HPV18 the cut off was 200 .

\section{Stool sample collection and examination}

Each study participant provided a stool sample of about a teaspoon full, which was collected on labelled polythene sheets about $10 \times 10 \mathrm{~cm}$. The stool samples were immediately processed and examined using the Kato-Katz method [15] for hookworms, Ascaris lumbricoides, Trichuris trichiura, and Schistosoma mansoni. For quality control, each stool sample was independently examined by two laboratory technicians within the school compound and in case of discrepant results, a third technician reviewed the sample. A sample was considered positive if ova or helminths were found, and negative if no ova or helminth were found. The students who had worms were informed about their results and encouraged to take treatment, which was provided within a month after enrolment as part of the national deworming exercise during Child Days Plus.

Serological evaluation of malaria antibodies (IgA, $\operatorname{Ig} G, \operatorname{Ig} M)$

Serological evaluation of malaria was done in the molecular laboratory of Makerere College of Health Sciences using a commercial malaria enzyme-linked immunosorbent assay (ELISA) kit (DRG Malaria ELISA EIA-4263 kit, DRG International, Inc., Springfield New Jersey, USA) according to the manufacturer's instructions. The kit uses four recombinant antigens to detect specific $\operatorname{IgG}, \operatorname{IgM}$, and IgA antibodies to Plasmodium falciparum ( $P$. faliciparum), Plasmodium vivax ( $P$. vivax), Plasmodium ovale ( $P$. ovale), and Plasmodium malariae (P. malariae). This enables the test to detect antibodies during all stages of infection. The antigens containing $P$. falciparum antigens (one merozoite surface antigen 1 (MSP1) and two merozoite surface antigen 2 (MSP2) with high sensitivity to antibodies against $P$. falciparum and different levels of crossreactivity to antibodies against other Plasmodium species.

Briefly, plastic wells were coated with a mixture of $P$. falciparum and $P$. vivax recombinant antigens. The 
antigenic similarity between Plasmodium species ensures that antibodies to all species can be detected. Specific antibodies in serum or plasma specimens combined with these antigens when a conjugate was added to a well in which the specimen has been incubated. After un-reacted material was removed by washing with wash buffer, the presence of bound enzyme indicated the presence of specific antibodies as revealed by a color change in the substrate/chromogen mixture. The intensity of the color was compared to that in the control wells to determine the presence or absence of a specific antibody, which was reported as positive or negative. The antibody malaria test is not part of the national guidelines (treatment based on blood smear results) for malaria management. Therefore, the results of the malaria test in this study were not used to suggest treatment.

\section{Data analysis}

The data were analysed using SPSS version 20. At univariate analysis, categorical data were summarized using percentages, while continuous variables were summarized by calculating the mean, standard deviation (SD), median, and range. MFI levels of antibodies to HPV-16/18 showed skewed distribution of the median antibody levels; therefore, the HPV antibody levels were transformed as $\log _{10}$ (MFI levels for antibody concentration). The mean of the MFI levels and corresponding 95\% confidence interval (CI) were calculated. The mean of the MFI values was compared between students who were exposed to helminths and/or malaria and those who were not. The Student's $t$-test was used to assess the difference between the MFI levels among students who showed evidence of exposure to helminths and/or malaria and those who were not exposed. The statistical level of significance was set at $\mathrm{p} \leq 0.05$. Box plots were used present the distribution of the mean MFI values for HPV-16/18 antibody levels among students exposed to helminths or malaria at baseline and those who were not.

\section{Results}

Study population

A total of 375 fully vaccinated students were present on the day of enrolment, of whom 211/375 $(56.3 \%)$ had parental consent and assented to participate in the study. The mean age of the students was 13.09 years of age (SD 1.48; range 10-16 years). At the 24-month post-vaccination follow-up visit, 195/211 (92.4\%) attended the visit. Sixteen students
$(16 / 211 ; 7.5 \%)$ were absent from the schools after repeated visits to the schools and were considered lost to follow-up.

\section{Malaria and helminth exposure at baseline (18 months post-vaccination)}

A total of 207/211 (98.1\%) students had adequate samples, and these were the ones who were included in the final analysis. There were 24/207 (6.8\%) students who were found to have evidence of helminth (hookworm, S. mansoni, and Trichuris trichiura) exposure in their stool by presence of ova or the actual helminth. Hookworm was more common 14/24 (58\%), followed by Trichuris trichiura $6 / 24$ (25\%), and $S$. mansoni 4/24 (16.6\%); none of the students was found to have A. lumbricoides. A total of 166/207 (80\%) were positive by the malaria parasite antibodies (IgA, IgG, IgM) test (Table 1).

\section{Immunogenicity}

All the students $(207 / 207 ; 100 \%)$ were seropositive for HPV-16 at month 18 (enrolment) and remained so through month 24 post-vaccination. However, for HPV-18, 205/207 (99.0\%) were seropositive at enrolment, and by month $24,188 / 191$ (98\%) were seropositive (two students who had initially been positive for HPV-18 at month 18 turned negative; one initially negative for HPV-18 remained negative through month 24 while the second one was lost to follow-up). Overall, the mean MFI HPV-16 antibody level at month 18 was 4,691 (95\% CI 4,438$4,958)$ and the mean MFI HPV-18 antibody response was $1,615(95 \%$ CI $1,469-1,776)$ with no significant change in mean MFI HPV-16 and 18 antibody level at month $24(\mathrm{n}=191)$ (Table 1).

The mean MFI HPV-16/18 antibody responses at month 18 were similar among students positive for any helminth and/or malaria exposure and those who were negative for both exposures (HPV-16 Table 1 and Figure 1; HPV-18 Table 1 and Figure 2). Further, at month 24, both mean MFI HPV-16 and mean MFI HPV-18 antibody titers were similar in both groups (Figures 3 and 4, respectively). However, there was a slight increase in the mean levels of HPV-18 antibodies at month 24 among students with evidence of malaria exposure. The difference was of borderline statistical significance: MFI unexposed, 1,166 (95\% CI 829-1,641); exposed 1,583 (95\% CI 1,391-1,802) $(\mathrm{p}=0.05)($ Table 1$)$. 
Table 1. Comparing overall $(n=207)$ mean MFI levels of HPV-16/18 antibodies of girls who were positive for helminths and malaria antibodies (IgA, IgG, IgM) at months 18 and 24 post-vaccination with those who were negative ${ }^{1}$

\begin{tabular}{|c|c|c|c|c|}
\hline & $\mathrm{N}$ & Mean MFI values $(95 \% \mathrm{CI})$ & Student's $t$-test & $P$ value \\
\hline \multicolumn{5}{|c|}{ Month 18} \\
\hline \multicolumn{5}{|c|}{ HPV-16 IgG } \\
\hline Overall & 207 & $4,691(4,438-4,958)$ & & \\
\hline \multicolumn{5}{|c|}{ Presence of any helminth } \\
\hline Yes & 14 & $4,075(2,949-5,632)$ & 1.35 & 0.18 \\
\hline No & 193 & $4,739(4,483-5,009)$ & & \\
\hline \multicolumn{5}{|c|}{ Malaria antibodies } \\
\hline Yes & 166 & $4,919(4,631-5,225)$ & -1.60 & 0.12 \\
\hline No & 41 & $4,366(3,625-5,258)$ & & \\
\hline \multicolumn{5}{|c|}{ HPV-18 IgG } \\
\hline Overall & 207 & $1,615(1,470-1,776)$ & & \\
\hline \multicolumn{5}{|c|}{ Presence of any helminth } \\
\hline Yes & 14 & $1,753(1,131-2,715)$ & -0.46 & 0.65 \\
\hline No & 193 & $1,816(1,456-1,770)$ & & \\
\hline \multicolumn{5}{|c|}{ Malaria antibodies } \\
\hline Yes & 166 & $1,747(1,563-1,952)$ & -1.57 & 0.12 \\
\hline No & 41 & $1,424(1,068-1,897)$ & & \\
\hline \multicolumn{5}{|c|}{ Month 24} \\
\hline \multicolumn{5}{|c|}{ HPV-16 IgG } \\
\hline Overall & 191 & $4,415(4,144-4,703)$ & & \\
\hline \multicolumn{5}{|c|}{ Presence of any helminth } \\
\hline Yes & 12 & $4,954(3,283-7,476)$ & -0.60 & 0.55 \\
\hline No & & $4,390(4,104-4,695)$ & & \\
\hline \multicolumn{5}{|c|}{ Malaria antibodies } \\
\hline Yes & 160 & $4,561(4,236-4,912)$ & -1.50 & 0.14 \\
\hline No & & $3,982(3,192-4,966)$ & & \\
\hline \multicolumn{5}{|c|}{ HPV-18 IgG } \\
\hline Overall & 191 & $1,453(1,306-1,617)$ & & \\
\hline \multicolumn{5}{|c|}{ Presence any helminth } \\
\hline Yes & 12 & $2,486(1,702-3,634)$ & -1.62 & 0.11 \\
\hline No & & $1,441(1,288-1,612)$ & & \\
\hline \multicolumn{5}{|c|}{ Malaria antibodies } \\
\hline Yes & 160 & $1,583(1,391-1,802)$ & -2 & 0.05 \\
\hline No & & $1,166(829-1,641)$ & & \\
\hline
\end{tabular}


Figure 1. Comparing MFI levels to HPV-16 antibodies at months 18 post-vaccination among students with and without helminth and malaria infection (IgA, IgG, IgM). There was no difference in the MFI levels to HPV-16 at 18 months postvaccination among the groups. The central line represents the median of the MFI levels; above and below the median line in the box are the 75th and 25th centiles; the whiskers represent the upper and lower MFI values and the dots represent the outlier value.

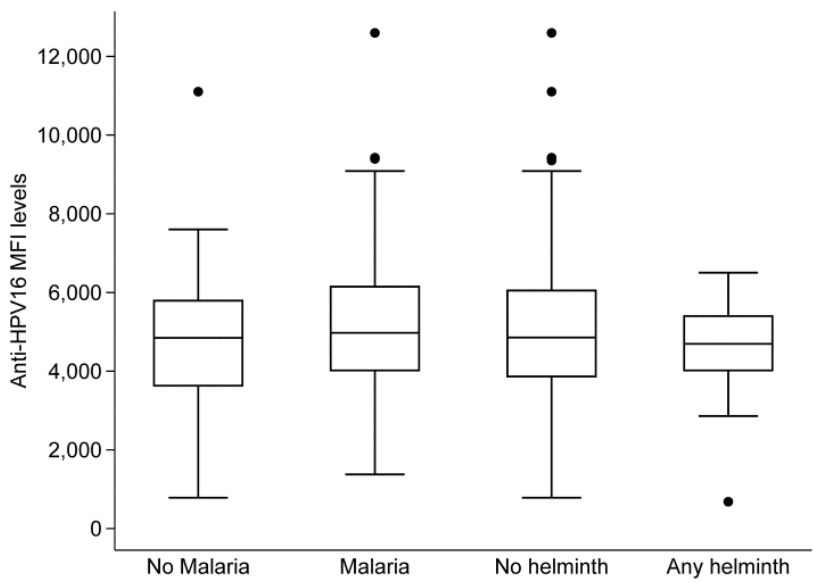

Figure 3. Comparing MFI levels to HPV-18 antibodies at months 18 post-vaccination among students with and without helminth and malaria infection (IgA, IgG, IgM). There was no difference in the MFI levels to HPV-18 at 18 months postvaccination among the groups. The central line represents the median of the MFI levels; above and below the median line in the box are the 75th and 25th centiles; the whiskers represent the upper and lower MFI values and the dots represent the outlier value.

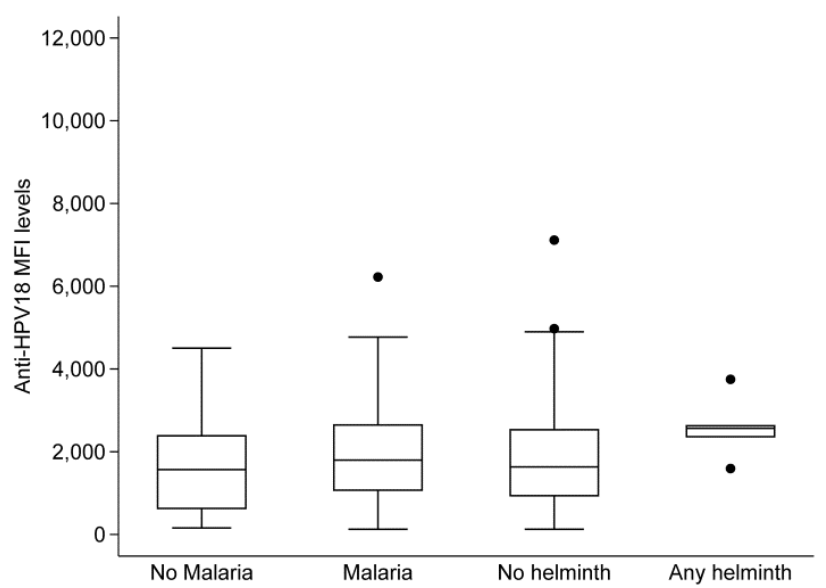

Figure 2.Comparing MFI levels to HPV-16 antibodies at months 24 post-vaccination among students with and without helminth and malaria infection (IgA, IgG, IgM). There was no difference in the MFI levels to HPV-16 at 24 months postvaccination among the groups. The central line represents the median of the MFI levels; above and below the median line in the box are the 75th and 25th centiles; the whiskers represent the upper and lower MFI values and the dots represent the outlier value.

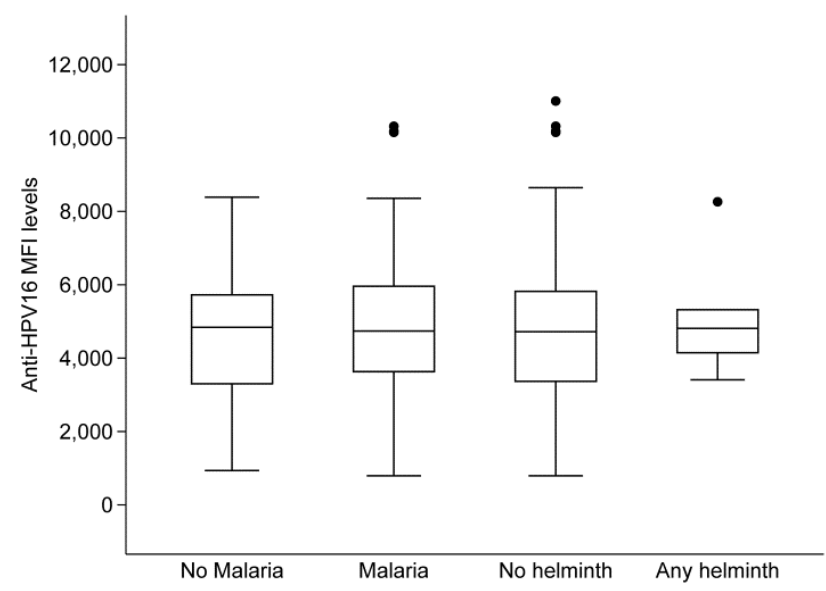

Figure 4. Comparing MFI levels to HPV-18 antibodies at months 24 post-vaccination among students with and without helminth and malaria infection (IgA, IgG, IgM). There was no difference in the MFI levels to HPV-18 at 24 post-vaccination among the groups. The central line represents the median of the MFI levels; above and below the median line in the box are the 75th and 25th centiles; the whiskers represent the upper and and lower MFI values and the dots represent the outlier value. There was a slight increase in the mean levels of HPV-18 antibodies at month 24 among students with evidence of malaria exposure. The difference was of borderline statistical significance $(\mathrm{p}=0.05)$.

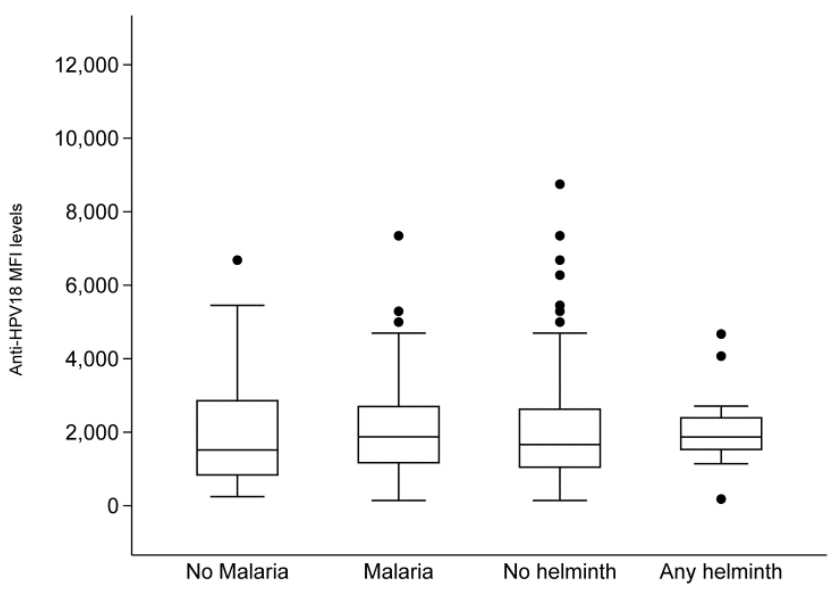


Table 2. Comparing mean MFI levels of HPV-16/18 antibodies among girls who were negative for either malaria antibodies (IgA, IgG, IgM) or helminth infestation and those who were positive to individual infestations at 18 month post-vaccination ${ }^{1}$

\begin{tabular}{|c|c|c|c|c|}
\hline & $\mathbf{N}$ & Mean MFI levels & Student's $t$-test & P value \\
\hline \multicolumn{5}{|l|}{ HPV-16 IgG } \\
\hline Not exposed to any infestation & 32 & 4,337 & & \\
\hline Exposed to only malaria & 156 & 4,952 & -1.7 & 0.09 \\
\hline \multicolumn{5}{|l|}{ HPV-18 IgG } \\
\hline Not exposed to any infestation & 32 & 1,420 & & \\
\hline
\end{tabular}

Furthermore, there was no difference in mean MFI level of HPV-16/18 antibodies at baseline between the students who had malaria and/or helminth exposure and those who were negative for both (Table 2).

\section{Ethical considerations}

The study was approved by the higher degrees research and ethics committee (HDREC) of Makerere University College of Health Sciences and by the Uganda National Council for Science and Technology (UNCST). Permission was sought from the local government in the district of study and school authorities. Consent from the parents/guardians of the students as well as assent from the students were obtained. The students who were found to have minor ailments such as ringworms, septic skin wounds, and menstrual period cramps were treated according to the treatment guidelines of the Uganda Ministry of Health. All the students were given deworming medication (mebendazole) within a month of enrolment as part of the routine national deworming exercise. The students who tested positive by the malaria parasites antibody (IgA, IgG, IgM) test were not treated for malaria because the malaria kit used in this study was the not the standard test for clinical malaria.

\section{Discussion}

This study evaluated the possible effect of malaria and helminth exposure on the immunogenicity of the bivalent HPV-16/18 vaccine among healthy adolescent students of unknown HIV serostatus in sub-Saharan Africa using a commercial multiplex serology testing kit. We found no negative effect on the immunogenicity to the bivalent HPV-16/18 vaccine among students who had evidence of exposure to malaria or helminths. This finding is similar to emerging evidence from a study of another African population in Tanzania [16]. In agreement with our study, the Tanzania study found no negative effect on the immune response to the bivalent HPV vaccine as well. In addition, at month 24 post-vaccination, we found that students with evidence of malaria exposure at month 18 had increased levels of antibodies to HPV-18, though it was of borderline statistical significance. This finding was similar to that seen in the Tanzania study above; however, the antibody increase was observed with HPV-16 and the mechanism remains unclear in both studies.

Though they had similar findings, the two studies had some differences in methodology. The period of enrolment post-vaccination when the blood and stool samples were collected was 7 months in the Tanzania study, and 18 months in our study. In addition, we used an antibody test as a marker for malaria exposure, whereas the Tanzania study tested for the presence of malaria parasitemia. However, both studies used the Kato-Katz method to detect helminths in the stool with some differences in the details of the procedures as well, including urine examination in the Tanzania study. Despite some of the methodological differences, the results of the two studies did not differ in terms of effect on the immunogenicity of the vaccine by malaria and helminth exposure. In our study, seropositivity to HPV-18 at baseline was less than $100 \%$ and had declined further at the 24-month follow-up visit. This observation of declining seropositivity to HPV-18 has been documented in other studies [17,18], though there were differences in the methods used to measure the antibodies.

Overall, the immunological response to the bivalent HPV-16/18 vaccine observed at month 18 was sustained through month 24 . This sustained level of antibody response from month 18 to 24 is similar to the documented trend whereby the antibodies were still sustained at up to 7.3 years of follow-up in one of the largest HPV clinical trials [18].

A strong sustained vaccine-induced immune response has been proven to protect against persistent 
HPV-16/18 and cervical intraepithelial neoplasia (CIN) disease [18-20]. We assume that this similar trend of sustained immune response observed in our study will translate into protection against CIN and eventually cervical cancer, though it will be many years before this is realized.

The study had some limitations. The students had already been vaccinated and were at month 18 postvaccination. We did not know when these students were exposed to these infections; in addition, a onepoint follow-up may not be representative of the whole effect on the general immunity. Only a small number of students had evidence of helminth infection, and therefore infections were not categorized into light, moderate, or heavy infection, a factor that has the potential to affect the antibody levels. The Kato-Katz method has low sensitivity in diagnosing helminths [21], coupled with the fact that it was done once in our study. In addition, we did not take stool samples at the second visit (24 months post-vaccination). After taking stool samples at 18 months post-vaccination, all the students were de-wormed and a repeat de-worming was undertaken shortly before our second visit of 24 months. Although our study showed no effect on the immune response by these co-pathogens, this data does not evaluate the effect of longer-term exposure or repeated exposures to these parasites on the immune response. Despite these limitations, however, this study still represents the day-to-day situation in routine HPV vaccination programs.

\section{Conclusions}

We found sustained high levels of antibodies to the bivalent HPV-16/18 vaccine up to 24 months postvaccination, and there was no difference in the antibody levels among the students with evidence of malaria and/or helminths exposure among adolescent students in Ibanda, a district of Uganda. Though longterm follow-up studies are needed, this data is reassuring and supports use of the prophylactic HPV$16 / 18$ vaccine in Africa.

\section{Acknowledgements}

This study was funded by the Uganda government through the Millennium Scientific Initiative project administered by the Uganda National Council of Science and Technology. More support was received from Makerere University staff development fund. We thank the study students and their parents/guardians. We also thank Dixon Matsiko and Robert Sserunjogi for the statistical support.

\section{References}

1. International Agency for Research on Cancer, World Health Organization (2013) GLOBOCAN 2012: Estimated Cancer Incidence, Mortality and Prevalence Worldwide in 2012. Lyon, France: IARC. Available: http://globocan.iarc.fr/Default.aspx. Accessed 7 November, 2014.

2. Walboomers JM, Jacobs MV, Manos MM, Bosch FX, Kummer JA, Shah KV, Snijders PJ, Peto J, Meijer CJ, Munoz N (1999) Human Papillomavirus is a necessary cause of invasive cervical cancer worldwide. J Pathol 189: 12-19.

1. 3. Banura C, Mirembe FM, Katahoire AR, Namujju PB, Mbonye AK, Mangen FW (2011) Epidemiology of HPV genotypes in Uganda and the role of the current preventive vaccines: A systematic review. Infect Agent Cancer 6: 11.

3. Paavonen J, Lehtinen M (2008) Introducing human papillomavirus vaccines - questions remain. Ann Med 40: 162-166.

4. Sabin EA, Araujo MI, Carvalho EM, Pearce EJ (1996) Impairment of tetanus toxoid-specific Th1-like immune responses in humans infected with Schistosoma mansoni. J Infect Dis 173: 269-272.

5. van Riet E, Hartgers FC, Yazdanbakhsh M (2007) Chronic helminth infestations induce immunomodulation: consequences and mechanisms. Immunobiology 212: 475490.

6. Elliott AM, Mawa PA, Webb EL, Nampijja M,Lyadda N,Bukusuba J, Kizza M,Namujju PB, Nabulime J, Ndibazza J, Muwanga M, Whitworth JA (2010) Effects of maternal and infant co-infections, and of maternal immunisation, on the infant response to BCG and tetanus immunisation. Vaccine 29: 247-255

7. Romanowski B (2011) Long term protection against cervical infection with the human papillomavirus: Review of currently available vaccines. Hum Vaccin 7: 161-169.

8. Cutts FT, Franceschi S, Goldie S, Castellsague X, de Sanjose S, Garnett G, Edmunds WJ, Claeys P, Goldenthal KL, Harper DM, Markowitz L (2007) Human papillomavirus and HPV vaccines: A review. Bull World Health Organ 85: 719-726.

9. Lehtinen M, Herrero R, Mayaud P, Barnabas R, Dillner J, Paavonen J, Smith PG (2006) Studies to assess the long-term efficacy and effectiveness of HPV vaccination in developed and developing countries. Vaccine 24 Suppl 3: S233-S241.

10. Nakalembe M, Banura C, Namujju BP, Mirembe MF (2014) The levels of anti-HPV16/18 and anti-HPV31/33/35/45/52/58 antibodies among AS04-adjuvanted HPV16/18 vaccinated and non-vaccinated Ugandan students aged 10-16 years. Infect Agent Cancer 9: 29.

11. Clifford GM, Shin HR, Oh JK, Waterboer T, Ju YH, Vaccarella S, Quint W, Pawlita M, Franceschi S (2007) Serologic response to oncogenic human papillomavirus types in male and female university students in Busan, South Korea. Cancer Epidemiol Biomarkers Prev 16: 1874-1879.

12. Namujju $P$, Waterboer $T$, Banura $C$, Muwonge $R$, Mbidde $E$, Byaruhanga R, Muwanga M, Surcel H, Pawlita M, Lehtinen M (2011) Risk of seropositivity to multiple oncogenichuman papillomavirus types among human immunodeficiency viruspositive and -negative Ugandan women. J Gen Virol 92: 2776-2783.

13. Waterboer T, Sehr P, Michael K, Franceschi S (2005) Multiplex human papillomavirus serology based on in situpurified glutathione s-transferase fusion proteins. Clin Chem51: 1845-1853. 
14. Katz N, Chaves A, Pellegrino J (1972) A simple device for quantitative stool thick smear technique in Schistosomiasis mansoni. Revista Do Instituto de Medicina Tropical de SaoPaulo 14: 397-400.

15. Brown J, Baisley K, Kavishe B, Changalucha J, Andreasen A, Mayaud P, Gumodoka B, Kapiga S, Hayes R, Watson-Jones $D$ (2014) Impact of malaria and helminth infections on immunogenicity of the human papillomavirus-16/18 AS04adjuvanted vaccine in Tanzania. Vaccine 32: 611-617.

16. Sow PS, Watson-Jones D, Kiviat N, Changalucha J, Mbaye KD, Brown J, Bousso K, Kavishe B, Andreasen A, Toure M, Kapiga S, Mayaud P, Hayes R, Lebacq M, Herazeh M, Thomas F, Descamps D (2013) Safety and immunogenicity of human papillomavirus-16/18 AS04-adjuvanted vaccine: a randomized trial in 10-25-year-old HIV-Seronegative African students and young women. J Infect Dis 207: 1753-1763.

17. De Carvalho N, Teixeira J, Roteli-Martins C, Naud P, De Borba P, Zahaf T, Sanchez N, Schuind A (2010) Sustained efficacy and immunogenicity of the HPV-16/18 AS04adjuvanted vaccine up to 7.3 years in young adult women. Vaccine 28: 6247-6255.

18. Paavonen J, Naud P, Salmeron J, Wheeler CM, Chow SN, Apter D, Kitchener H, Castellsague X, Teixeira JC, Skinner SR, Hedrick J, Jaisamran U, Limson G, Garland S, Szarewski A, Romanowski B, Aoki FY, Schwarz TF, Poppe WA, Bosch FX, Jenkins D, Hardt K, Zahaf T, Descamps D, Struyf F, Lehtinen M, Dubin G (2009) Efficacy of human papillomavirus (HPV)-16/18 AS04-adjuvanted vaccine against cervical infection and precancer caused by oncogenic HPV types (PATRICIA): final analysis of a double-blind, randomised study in young women. Lancet 374: 301-314.

19. Harper DM, Franco EL, Wheeler CM, Moscicki AB, Romanowski B, Roteli-Martins CM, Jenkins D, Schuind A, Costa Clemens SA, Dubin G (2006) Sustained efficacy up to 4.5 years of a bivalent L1 virus-like particle vaccine against human papillomavirus types 16 and 18: follow-up from a randomised control trial. Lancet 367: 1247-1255.

20. Booth M, Vounatsou P, N'goran E, Tanner M, Utzinger J (2003) The influence of sampling effort and the performance of the Kato-Katz technique in diagnosing Schistosoma mansoni and hookworm co-infections in rural Côte d'Ivoire. Parasitology 127: 525-531.

\section{Corresponding author}

Dr. Nakalembe Miriam

Department of Obstetrics and Gynaecology, School of Medicine

Makerere College of Health Sciences

PO BOX 7072, Kampala, Uganda

Phone: +256414534361

Fax: +256414533451

Email: ivuds@yahoo.com

Conflict of interests: No conflict of interests is declared. 